\title{
A new carbon phase with direct bandgap and high carrier mobility as electron transport material for perovskite solar
} cells

\author{
Ping-Ping Sun ${ }^{1}$, Lichun Bai ${ }^{1,2}$, Devesh R. Kripalani ${ }^{1}$ and Kun Zhou ${ }^{1,2}$
}

Rapid development of perovskite solar cells is challenged by the fact that current semiconductors hardly act as efficient electron transport materials that can feature both high electron mobility and a well-matched energy level to that of the perovskite. Here we show that T-carbon, a newly emerging carbon allotrope, could be an ideal candidate to meet this challenge. By using first-principles calculations and deformation potential theory, it is found that T-carbon is a semiconductor with a direct bandgap of $2.273 \mathrm{eV}$, and the energy level in the conduction band is lower than that of perovskite by $0.5 \mathrm{eV}$, showing a larger force of electron injection. Moreover, the calculated electron mobility can reach up to $2.36 \times 10^{3} \mathrm{~cm}^{2} \mathrm{~s}^{-1} \mathrm{~V}^{-1}$, superior to conventional electron transport materials such as $\mathrm{TiO}_{2}, \mathrm{ZnO}$ and $\mathrm{SnO}_{2}$, which will facilitate more efficient electron separation and more rapid diffusion away from their locus of generation within the perovskite absorbers. Furthermore, the bandgap of T-carbon is highly sensitive to strain, thus providing a convenient method to tune the carrier transport capability. Overall, T-carbon satisfies the requirements for a potential efficient electron transport material and could therefore be capable of accelerating the development of perovskite solar cells.

npj Computational Materials (2019)5:9; https://doi.org/10.1038/s41524-018-0146-z

\section{INTRODUCTION}

In recent years, organic and inorganic hybrid perovskite solar cells (PSCs), a highly efficient newcomer to the solar cell family, has emerged with great commercial potential to become a leader of next-generation solar energy photovoltaic technology. ${ }^{1-3}$ The power conversion efficiency (PCE) of PSCs has reached a certified value of $22.7 \%$ from $3.8 \%$ in a short span of nine years. ${ }^{4,5}$ PSCs mostly adopt the configuration of transparent electrode/electron transport material (ETM)/perovskite/hole transport material (HTM)/ metal electrode. To increase the $P C E$, it is essential to precisely manipulate the carriers along the entire pathway from the perovskite absorber to both electrodes. The ETM is one of the most important components affecting the photovoltaic performance of PSCs by controlling the carrier concentration. A favorable alignment between the ETM and perovskite allows for the efficient extraction of photogenerated carriers without inducing excessive interface recombination.

At present, $\mathrm{TiO}_{2}$ is the most common and efficient material used as the ETM in PSCs to collect and transport electrons from the perovskite absorber layer. ${ }^{6,7}$ Nevertheless, although the electron injection rates from the perovskite absorber to $\mathrm{TiO}_{2}$ ETM are very fast, the electron recombination rates are also very high due to the low electron mobility. Furthermore, the production of conductive crystalline $\mathrm{TiO}_{2}$ requires relatively high sintering temperatures $\left(400-500^{\circ} \mathrm{C}\right)$. These disadvantages limit its application widely. ${ }^{8}$ Therefore, the design of a new and efficient ETM is crucial and imperative for the development of PSCs. An excellent ETM should possess two fundamental properties, where on the one hand, the material should possess high electron mobility to fulfill the requirement for fast electron transport while avoiding interfacial electron recombination, on the other hand, its energy level is supposed to be well-matched to that of the perovskite to facilitate electron injection and hole blocking simultaneously. ${ }^{9}$ Even now, many new ETMs have been reported, including inorganic materials $\mathrm{ZnO}_{1}{ }^{10} \mathrm{SnO}_{2},{ }^{11}$ WO $x,{ }^{12}$ organic materials $\mathrm{C}_{60},{ }^{13}$ PC61BM, PC71BM, ${ }^{14,15}$ and so on. However, the limited improvement on the carrier mobility has not achieved the expectation desired for photovoltaic performance.

Here we propose a new potential ETM candidate, T-carbon, which was initially theoretically predicted to be structurally stable and have excellent electronic properties for wide potential applications by Su et al. ${ }^{16}$ in 2011. Recently, Zhang et al. ${ }^{17}$ successfully turned the prophecy into reality by means of pseudotopotactic conversion, where T-carbon can be produced from carbon nanotubes under picosecond laser irradiation in methanol. Moreover, the optical absorption measurements and rough calculation indicate that bulk T-carbon is a semiconductor with a direct bandgap of about 3-5 eV. Up to now, the carrier transport behavior of T-carbon has not been reported in theory and experiment.

Thus, in this work, by combining first-principles calculations and deformation potential (DP) theory, ${ }^{18}$ the electronic structures and intrinsic mobility of bulk T-carbon are systematically investigated. The theoretical evidence shown in this study demonstrates that Tcarbon is a semiconductor with a desirable moderate direct bandgap of around $3 \mathrm{eV}$, and the band structure can be modified by the strain effect. Its conduction band energy level matches well with that of the perovskite, giving it a better advantage in extracting the electron injection from the generation location to

\footnotetext{
${ }^{1}$ School of Mechanical and Aerospace Engineering, Nanyang Technological University, 50 Nanyang Avenue, Singapore 639798, Singapore and ${ }^{2}$ SMRT-NTU Smart Urban Rail Corporate Laboratory, Nanyang Technological University, 50 Nanyang Avenue, Singapore 639798, Singapore

Correspondence: Kun Zhou (kzhou@ntu.edu.sg)
}

Received: 18 July 2018 Accepted: 13 December 2018

Published online: 15 January 2019 
the ETM layer. In addition, T-carbon possesses better carrier transport properties compared with traditional semiconductor materials, especially with respect to its electron transport performance, where its electron mobility can reach up to $2.36 \times$ $10^{3} \mathrm{~cm}^{2} \mathrm{~s}^{-1} \mathrm{~V}^{-1}$. The excellent properties discovered for T-carbon renders it to be an effective and appropriate candidate as the ETM for high-performance PSCs.

\section{RESULTS}

\section{Electronic properties}

In this work, the geometric structure of T-carbon is optimized with the Perdew-Burke-Ernzerhof (PBE) method combined with vdW interactions. The detailed discussion on the lattice constants and geometry are shown in Note S2 in Supplementary Information (SI). Our results illustrate that the calculated lattice constants are in good agreement with experimental results, indicating that the method adopted is reliable.

The structural stability of T-carbon is also investigated by considering the effect of thermal fluctuation. The root-meansquare deviation (RMSD) and root-mean-square fluctuation (RMSF) are calculated by molecular dynamics and the results indicate that T-carbon can show good stability in the temperature range of $100-340 \mathrm{~K}$, which accords with the temperature of perovskites used in PSCs (further discussions are given in Note S2 and Figure S1).

Based on the optimized configuration, the band structures of Tcarbon are predicted with PBE, PBEsol, ${ }^{19}$ and HSE06 functionals ${ }^{20}$ as well as with different vdW interaction methods including optB86b-vdW, ${ }^{21}$ optB88-vdW, ${ }^{22}$ vdW-DF2, ${ }^{23}$ DFT-D2. ${ }^{24}$ The detailed results of the band structures from the above methods are discussed in Note S3. As shown in Fig. S2 and Table S2, it is found that all methods exhibit a direct bandgap character, with PBE and PBEsol results giving similar bandgaps. Compared with PBE and PBEsol, HSE06 show larger bandgaps. Previous reports have pointed out that PBE and PBEsol functionals tend to underestimate the bandgap of semiconductors, while the hybrid HSE06 functional can give more accurate results, which are comparable with experimental data. ${ }^{25,26}$ Moreover, the optB86bvdW functional can give further improvement in the description of the binding energy. ${ }^{27}$ Hence, HSE06 combined with the optB86b-
vdW functional is the best selection for the description of electronic properties, where T-carbon possesses a direct bandgap of $2.273 \mathrm{eV}$. Furthermore, the band structures near the valence band maximum (VBM) or the conduction band minimum (CBM) exhibit remarkable in-plane dispersion behavior in the $\Gamma$ to $M$ or $\Gamma$ to $\mathrm{R}$ direction in the Brillouin zone, which suggests that bulk Tcarbon may have relatively high carrier mobilities.

The computed band structure based on the HSE06-optB86bvdW functional is shown in Fig. 1a, from which the orbital charge density on different conduction and valence bands are analyzed (Fig. 1b). In the conduction bands CB1-CB3, the charge densities are mainly located in the inter-tetrahedron bonds of the structure, while in the valence bands VB1-VB3, the charge densities are mainly distributed in the tetrahedrons of the structure. Once the atoms in T-carbon experience electronic excitation, appreciable electrons will leap from the valence bands to the conduction bands, gathering holes within the valence bands. In the excitation process, the electrons transfer from the original intra-tetrahedron bonds to inter-tetrahedron bonds, making the latter have much higher electron densities with shorter bond characteristics. Therefore, the inter-tetrahedron bonds are relatively stronger than intra-tetrahedron bonds with a better ability to accumulate electrons. The differences in bond lengths play a crucial role in achieving structural stability by balancing the strain from adjacent tetrahedrons.

The corresponding electrostatic potential contour plots of VB3-CB3 (Fig. S5-S10) show that the electrons are densely localized around the intra-tetrahedron bonds and display a strong covalent bonding feature. However, there is almost no electron localization in the inter-tetrahedron bonds, which reflect their ionic nature. The interlaced bonding features manifest that each tetrahedron connects with each other through ionic bonds. This special structural character of T-carbon will make the geometry more stable and is favorable for promoting electron transfer from intra-tetrahedron bonds to inter-tetrahedron bonds.

The three-dimensional contour plots of the VBM and CBM show different distributions and positions of peaks (Fig. 2a). The peaks where electrons accumulate uniformly and locally around the surface in the CBM reflect antibonding states in the structure, while the conjoint peaks in the VBM means that there exist strong bonding states. Both kinds of peak distributions correspond to the (a)

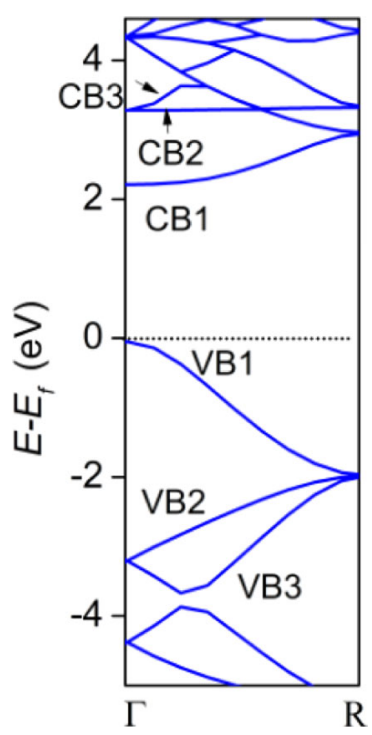

(b)
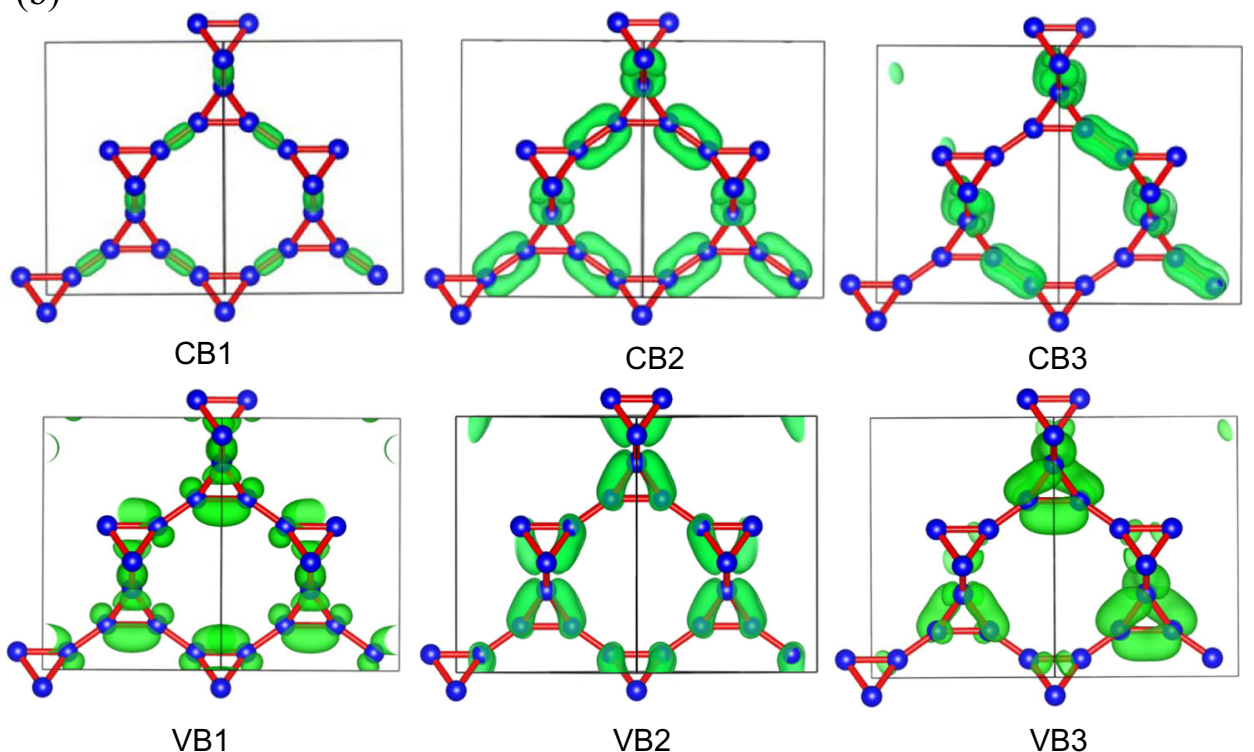

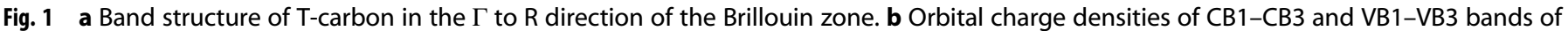
T-carbon 
(a)

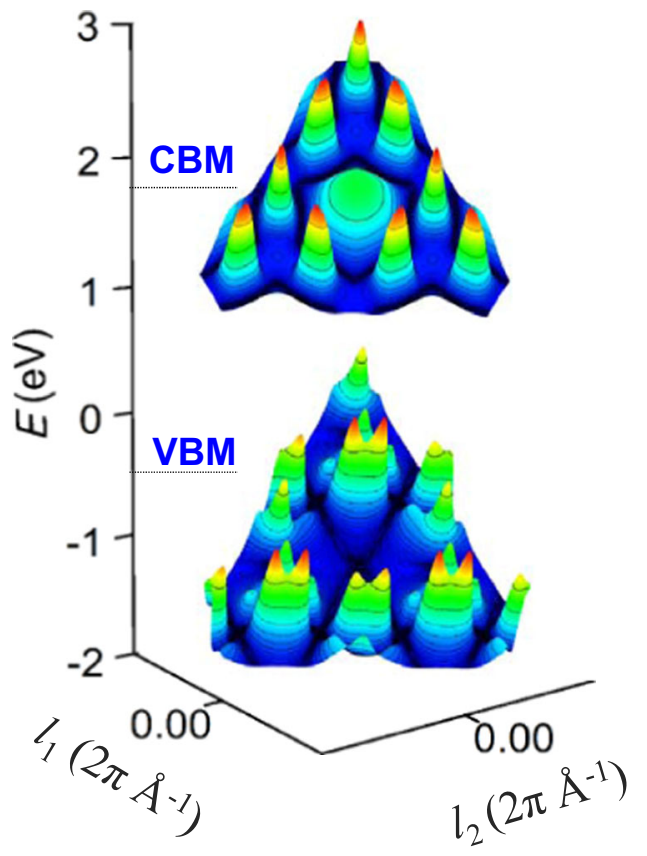

(b)

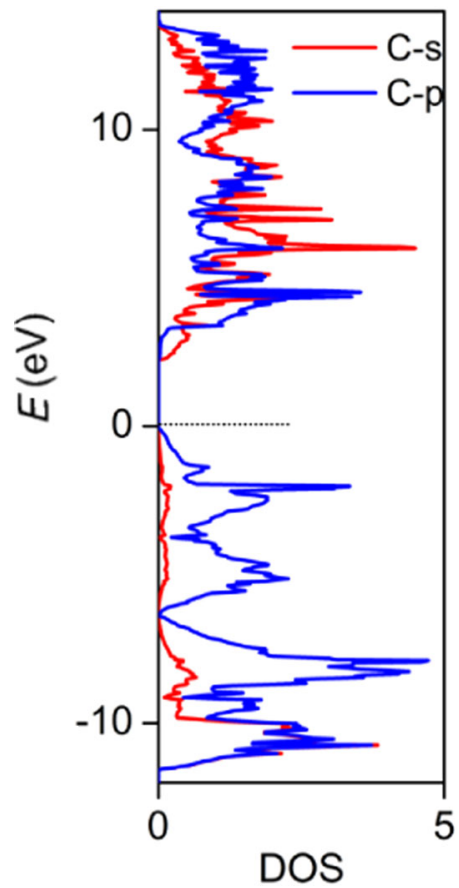

Fig. 2 a Three-dimensional electrostatic potential contour plots of CBM and VBM and $\mathbf{b}$ the PDOS structure of T-carbon

charge densities located in the CBM and VBM as shown in Fig. 1b. The different distributions in the contour plots shown in both the CBM and VBM reveal a distinct anisotropic behavior near the $\Gamma$ point for both electrons and holes.

A further DOS calculation (Figs. S11-S14) reveals that T-carbon possesses a distinct semiconductor character with strong orbital density in the conduction bands and relatively weak density in the valence bands. Closer analyses of the electronic orbitals illustrated by the projected density of states (PDOS; Fig. 2b) show that the conduction bands are dominated by $C 2 s$ and $2 p$ orbitals, whereas the valence bands are mainly formed by $C 2 p$ orbitals with a rather weak contribution from $C 2 s$ orbitals. Besides, the high hybridization and overlap between $s$ and $p$ orbitals from $3 \mathrm{eV}$ to the top of the conduction band show a strong interactive movement of the excited electrons in the conduction bands, where electrons will transfer from the lower to higher energy state via the interactive transmission between the different orbitals. This transmission can lead to anisotropic charge distribution in the energy states where the charge densities are not homogeneously distributed on the intra-tetrahedron and inter-tetrahedron bonds, as shown in Fig. 1b. Similar orbital hybridization can also be seen in the valence bands. Nevertheless, there is no hybridization at both the top of the valence bands and bottom of the conduction bands, where are singly composed of $2 p$ and $2 s$ orbitals, respectively. In the band structure, the bandgap is formed between bonding $(\sigma)$ and antibonding $\left(\sigma^{*}\right)$ orbitals located in the VBM and CBM, respectively. The direct bandgap at the $\Gamma$ point means that the $\sigma$ and $\sigma^{*}$ orbitals are derived from the contributions of $2 p$ and $2 s$ orbitals, respectively. Additionally, the band edges are relatively dispersed in the vicinity of the $\Gamma$ point, indicative of good carrier transport mobilities. $^{28}$

In efficient PSCs, the CBM of the ETM should be lower compared with that of the perovskite to achieve fast electron injection. Previous work has shown that suitable energy level offset can promote the efficient injection of electrons from the perovskite to
ETM. $^{29-32}$ The energy level arrangements of T-carbon, popular ETMs $\left(\mathrm{TiO}_{2}, \mathrm{SnO}_{2}\right.$, and $\left.\mathrm{ZnO}\right)$ and frequently used perovskites are provided in Fig. 3a. In the diagram, the CBM energy level of Tcarbon is $-4.4 \mathrm{eV}$, close to that of both $\mathrm{SnO}_{2}$ and $\mathrm{ZnO}$ which have been testified to yield quicker charge injection rates as compared to $\mathrm{TiO}_{2}{ }^{9,33-35}$ The similar energy level offset as with $\mathrm{SnO}_{2}$ and $\mathrm{ZnO}$ indicates that $\mathrm{T}$-carbon may also have faster electron injection rate than $\mathrm{TiO}_{2}$, and will thus facilitate more efficient electron acceptance and more rapid diffusion away from their locus of generation, thereby effectively retarding geminate recombination.

As an advantage, the ETM, similar to HTM, is supposed to be colorless, because the ultraviolet-visible (UV-Vis) light absorption of the ETM would affect the light-harvesting efficiency of the perovskites. ${ }^{36}$ The optical absorption of T-carbon given by the HSE06-optB86b-vdW functional is shown in Fig. 3b. It is found that T-carbon shows strong absorption in the ultraviolet range of $180-280 \mathrm{~nm}$. In addition, a cluster model containing 200 atoms (Fig. 3c) based on the TD-B3LYP/6-31G level using Gaussian 16 code is also calculated, ${ }^{37}$ for which the maximum absorption is at $249 \mathrm{~nm}$ (Fig. 3d), where the excitation energy is $4.94 \mathrm{eV}$. The dominant transition is from the highest occupied molecular orbital (HOMO) to the lowest unoccupied molecular orbital (LUMO). The HOMO is mainly localized on the center of T-carbon, while the LUMO is located close to the edge. Both the two calculations manifest that there is no absorption in the visible region. For the classic $\mathrm{TiO}_{2}$ ETM, it has strong absorption at short wavelengths in the range of $300-400 \mathrm{~nm}^{38,39}$ The optical absorption of common perovskites are in the range of $300-800 \mathrm{~nm} .^{40,41}$ The overlapping absorption in the UV-Vis region between $\mathrm{TiO}_{2}$ and perovskite will promote competition for the extraction of photogenerated electrons. Compared with $\mathrm{TiO}_{2}$, the optical calculation of $\mathrm{T}$ carbon favorably highlights its preferential role as the ETM as it will not affect the absorption of perovskite materials in the visible spectrum. 
(a)

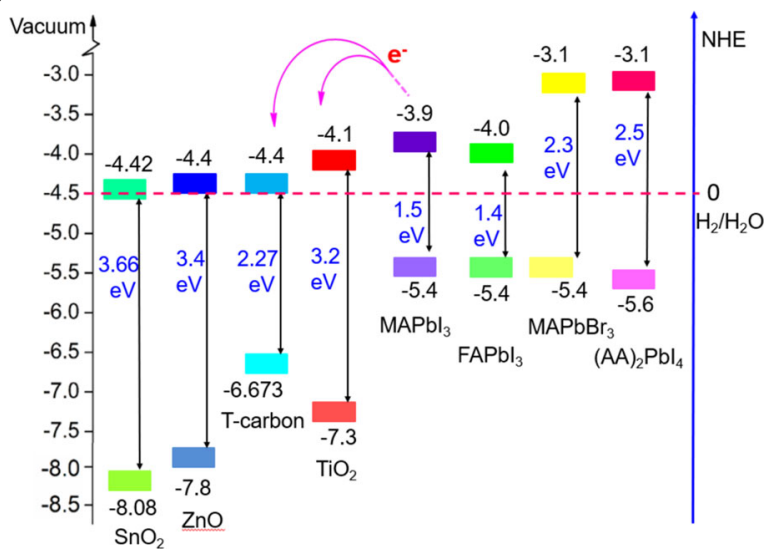

(c)

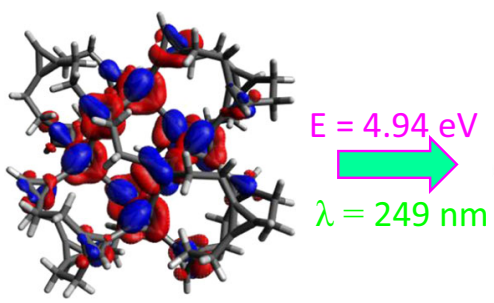

HOMO

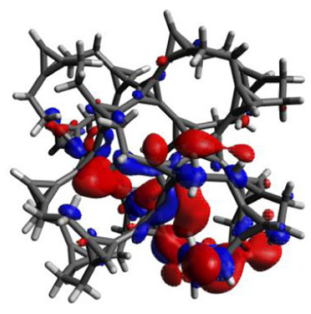

LUMO (b)

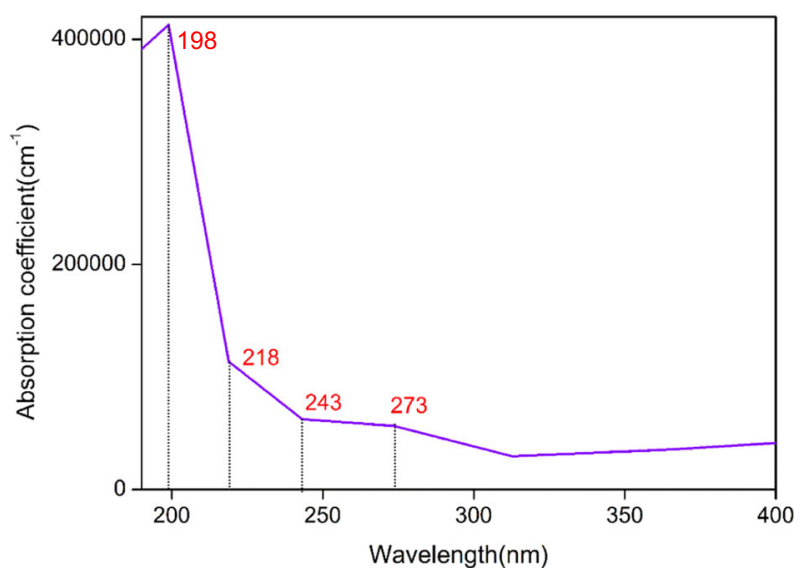

(d)

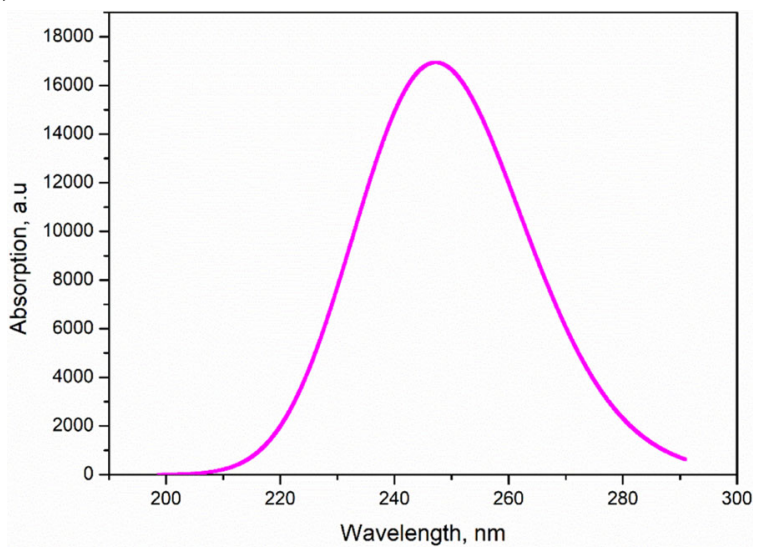

Fig. 3 a Energy levels of T-carbon compared with those of $\mathrm{SnO}_{2}, \mathrm{ZnO}, \mathrm{TiO}_{2}, \mathrm{MAPbl}_{3}, \mathrm{FAPbl}_{3}, \mathrm{MAPbBr}_{3}$, and $(\mathrm{AA})_{2} \mathrm{Pbl}_{4}$. The blue data refer to their corresponding bandgaps. b The absorption spectra of T-carbon calculated at HSE06-optB86b-vdW level. c The HOMO and LUMO of a cluster model of T-carbon containing 200 atoms calculated at TD-B3LYP/6-31G level. $E$ denotes the excitation energy and $\lambda$ is the maximum absorption wavelength. $\mathbf{d}$ The absorption spectra of T-carbon cluster

\section{Carrier mobility}

For potential ETMs, aside from having a matched energy level, a reasonable high carrier mobility is another indispensable factor. Previous reports have verified that the high electron mobility of ETMs will facilitate fast extraction and transport photogenerated electrons from perovskites to ETMs, contributing towards improved $J_{\mathrm{Sc}}$ and high efficiency with negligible $J-V$ hysteresis. s, $30,31,33,42$ For example, PSCs based on $\mathrm{SnO}_{2}$ or $\mathrm{ZnO}$ with suitable energy levels and high electron mobility have demonstrated excellent photovoltaic properties. . $^{92,33}$

As shown in Table S3, the calculated carrier mobilities of black phosphorus, $\mathrm{MoS}_{2}$, graphene, etc. based on DP theory are in the same order of magnitude as their experimental data, which indicates that the method we adopt here to calculate the carrier mobilities of T-carbon is reliable.

As shown in Eq. (1), the effective mass has an important effect on the carrier mobility. The calculated effective masses $m^{*}$ are listed in Table 1. Due to the intrinsic cubic structure, $m_{x}=m_{y}=m_{z}$, T-carbon shows an isotropic transport character. The calculated electron and hole effective masses are $0.22 \mathrm{~m}_{0}$ and $0.66 \mathrm{~m}_{0}$, respectively, which are even comparable to those of perovskite $\mathrm{MAPbl}_{3} / \mathrm{MASnl}_{3}\left(0.2-1.3 m_{0}\right){ }^{43}$ Besides, the hole effective mass is three times larger than that of electrons, which means that the electron transport ability of T-carbon is better than its hole transport ability.
Table 1. Calculated effective mass $\left(m^{*}\right)$ for electrons and holes along the $\Gamma$ to $\mathrm{R}$ direction, deformation potential constant $\left(E_{1}\right), 3 \mathrm{D}$ elastic constant $\left(C^{3 \mathrm{D}}\right)$, carrier mobility $(\mu)$, and relaxation-time $(\tau)$ along the transport direction of T-carbon at $300 \mathrm{~K}$

\begin{tabular}{llllll}
\hline Carrier type & $m^{*} / m_{0}$ & $E_{1}(\mathrm{eV})$ & $C^{3 \mathrm{D}}\left(10^{9} \mathrm{~N} \mathrm{~m}^{-2}\right)$ & $\mu\left(10^{3} \mathrm{~cm}^{2} \mathrm{~s}^{-1} \mathrm{~V}^{-1}\right)$ & $\tau$ (fs) \\
\hline Electron & 0.22 & -14.88 & 186.2 & 2.36 & 290.13 \\
Hole & 0.66 & -10.89 & 186.2 & 0.27 & 102.21
\end{tabular}

Besides the effective mass, the carrier mobility is also strongly influenced by the 3D elastic constant $C^{3 \mathrm{D}}$ and DP constant $E_{1}$. To compute $C^{3 D}$ and $E_{1}$, the lattice of the cell has been dilated and compressed by $1 \%$ along the transport direction. The total energies and positions of the VBM and CBM are calculated with respect to the degree of dilation and compression. All atomic positions are fully relaxed at each dilation and compression step. The electronic energies are obtained at the HSE06-optB86b-vdW level. Figure 4a shows the variation of total energy with applied uniaxial strain along the lattice direction and $C^{3 \mathrm{D}}$ can be obtained by fitting the energy vs strain curve. Figure $4 \mathrm{~b}$ depicts the shifts of both band edges VBM and CBM as a function of uniaxial strain, where $E_{1}$ can be calculated from the slope of the fitted lines. Based on the obtained $m^{*}, C^{3 D}$, and $E_{1}$, the calculated electron mobility is found to be $2.36 \times 10^{3} \mathrm{~cm}^{2} \mathrm{~s}^{-1} \mathrm{~V}^{-1}$, which is about 10 times higher 
(a)

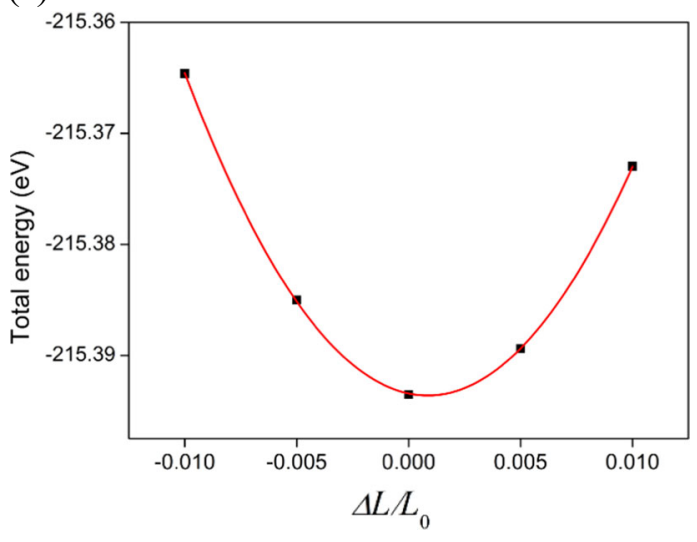

(b)

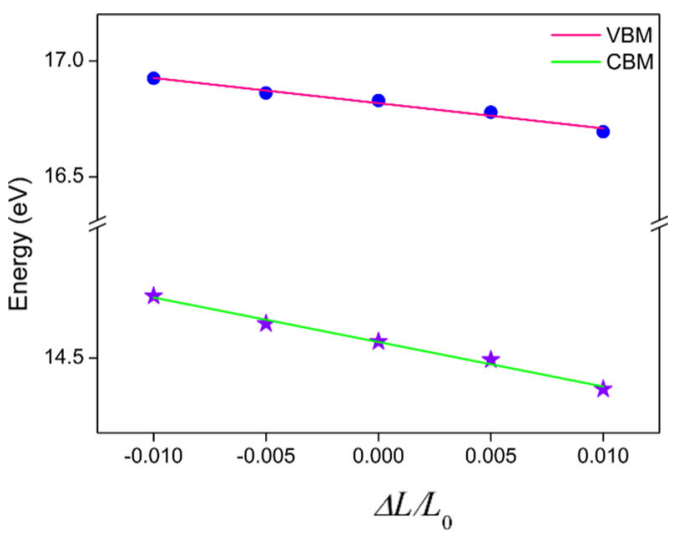

Fig. 4 a The relationship between total energy and lattice dilation along the transport direction. $\mathbf{b}$ Band energy shifts of VBM and CBM under uniaxial strain along the lattice dilation direction

than that of holes $\left(2.7 \times 10^{2} \mathrm{~cm}^{2} \mathrm{~s}^{-1} \mathrm{~V}^{-1}\right)$. As the strain range is widened from $\pm 1 \%$ to $\pm 2 \%, \pm 3 \%, \pm 4 \%$ and $\pm 5 \%$, respectively, the variation of carrier mobility mainly depends on changes in $C^{3 \mathrm{D}}$ and $E_{1}$ (Fig. S16 and Table S4). At each strain window setting, the ratio of electron to hole mobility retains the same value of about 10 . Carrier mobility first decreases for the strain range of $\pm 2 \%$ before increasing as the strain range is widened from $\pm 3 \%$ to $\pm 5 \%$, yet still remaining low with respect to the initial strain range of $\pm 1 \%$. In view of the changes in carrier mobility subject to tensile strain, we find that the high electron mobility of T-carbon mainly originates from the low electron effective mass $m_{\mathrm{e}}^{*}$.

It is worth to mention that the predicted carrier mobilities of Tcarbon are comparable and even higher than those of many current popular 2D materials, traditional semiconductor materials, and perovskites. For example, the electron mobility of T-carbon is distinctly higher than that of various popular 2D materials and semiconductor materials, such as black phosphorus $\left(10^{3} \mathrm{~cm}^{2} \mathrm{~s}^{-1}\right.$ $\left.\mathrm{V}^{-1}\right){ }^{44} \mathrm{TIO}_{2}\left(3.3 \times 10^{3} \mathrm{~cm}^{2} \mathrm{~s}^{-1} \mathrm{~V}^{-1}\right){ }^{45} \mathrm{Si}\left(1.5 \times 10^{3} \mathrm{~cm}^{2} \mathrm{~s}^{-1} \mathrm{~V}^{-1}\right){ }^{46}$ and $\mathrm{TiO}_{2}\left(5.24 \times 10^{-2} \mathrm{~cm}^{2} \mathrm{~s}^{-1} \mathrm{~V}^{-1}\right){ }^{47}$ For hole transport performance, Tcarbon reveals significant advantage compared with $\mathrm{MoS}_{2}$ $\left(200 \mathrm{~cm}^{2} \mathrm{~s}^{-1} \mathrm{~V}^{-1}\right)_{1}^{48}$ perovskite $\mathrm{MAPbl}_{3}\left(1-5 \mathrm{~cm}^{2} \mathrm{~s}^{-1} \mathrm{~V}^{-1}\right)_{1}^{49,50}$ and $\mathrm{TiO}_{2}\left(0.16 \mathrm{~cm}^{2} \mathrm{~s}^{-1} \mathrm{~V}^{-1}\right) .^{47}$ In sum, compared with currently available materials, T-carbon is prospected to have strong transmitting capacity, giving that its high electron mobility can lead to further improved short-circuit current density $\left(J_{\mathrm{SC}}\right)$ and efficiency. ${ }^{51}$ Considering the highly effective performance of T-carbon, it is anticipated to be a viable replacement for traditional $\mathrm{TiO}_{2}$ as the ETM.

In addition, the strain effect on the electronic properties of Tcarbon (Fig. 5 and Fig. S17) is examined. For both tensile and compressive strain, T-carbon preserves its direct bandgap character. The bandgap increases linearly from $-5 \%$ to $-2 \%$ and from -1 to $0 \%$ strain, beyond which it then increases in a soft growth way up to $5 \%$ strain where it has a slightly lower slope than that during the compressed phases. Along with the variation of strain from -1 to $1 \%$, the bandgap fluctuates in the range of -57 to $24 \mathrm{meV}$ relative to that of the equilibrium structure. Interestingly, $1 \%$ compressive strain has a larger effect on the bandgap variation than $1 \%$ tensile strain, which means that the compressive treatment has a greater effect on the electronic property of Tcarbon than the tensile stretch. But when the applied strain is higher than $2 \%$, the tensile stretch has larger effect than the compressive treatment as the bandgap can increase by up to $305 \mathrm{meV}$ with respect to the pristine bandgap. The bandgap evolution under applied strain, as shown in Fig. $5 f$ and Fig. S17i, reflects that T-carbon will show an increasing bandgap with tensile strain and a declining bandgap with compressive strain.
This may provide a new perspective for the development of new functionalities for T-carbon, which can show a tunable band structure according to the different performance evolving from tensile and compressive strain.

\section{DISCUSSION}

In conclusion, we report a new carbon allotrope as the ETM in PSCs. The electronic properties and carrier mobility of T-carbon are investigated by first-principles methods and DP theory. The results indicate that T-carbon is a natural semiconductor with a direct bandgap of $2.273 \mathrm{eV}$. The conduction band energy level matches well with that of perovskites, which is in favor of electron injection from the point of generation into the ETM layer. Besides, its optical absorption in the ultraviolet range will not compete against the light harvesting capacity of perovskites in the UV-Vis region. It is more important that T-carbon possesses a high electron mobility of $2.36 \times 10^{3} \mathrm{~cm}^{2} \mathrm{~s}^{-1} \mathrm{~V}^{-1}$, which is superior to that of familiar ETMs such as $\mathrm{TiO}_{2}, \mathrm{ZnO}, \mathrm{SnO}_{2}$, and even $\mathrm{MAPbl}_{3}$ perovskite. These features portend that T-carbon will be an excellent ETM candidate for high-performance PSCs. The different band structures that evolve from tensile and compressive strain suggest that imposed strain can be an effective approach to modulate its transport properties. Based on its special structure and high potential applications, it will be intriguing to embark on experimental investigations to realize these predictions for a variety of practical applications.

\section{METHODS}

Density functional theory calculations

For T-carbon, geometry optimization and electronic properties calculations are carried out using density functional theory (DFT) methods within the generalized gradient approximation and PBE exchange correlation functional, as performed in the Vienna ab initio simulation package (VASP). ${ }^{52}$ The ion-electron interactions are described by the projected augmented wave method, ${ }^{53}$ and the energy cut-off for the plane wave basis is set to $500 \mathrm{eV}$. Periodic boundary conditions are applied to all systems. For geometry optimization, a $6 \times 6 \times 6$ Monkhorst Pack grid is adopted, and all the structures are allowed to relax during this process with a conjugate gradient algorithm until the energy on the atoms is less than $1.0 \times 10^{-5} \mathrm{eV}$. The maximum force allowed on each atom is $0.01 \mathrm{eV}$ $\AA^{-1}$. Since the van der Waals (vdW) interactions have a great impact on the geometry and electronic properties, ${ }^{54,55}$ different vdW correction methods such as the optB86b exchange functional (optB86b-vdW), ${ }^{21,22}$ optB88 exchange functional (optB88-vdW), ${ }^{22}$ vdW-DF2 exchange functional ${ }^{23}$ as well as the DFT-D2 approach proposed by Grimme ${ }^{24}$ are considered here to accommodate the $\mathrm{vdW}$ interactions. For electronic structure calculations, a fine $8 \times 8 \times 8$ grid mesh is used. Since the PBE functional has been 
(a)

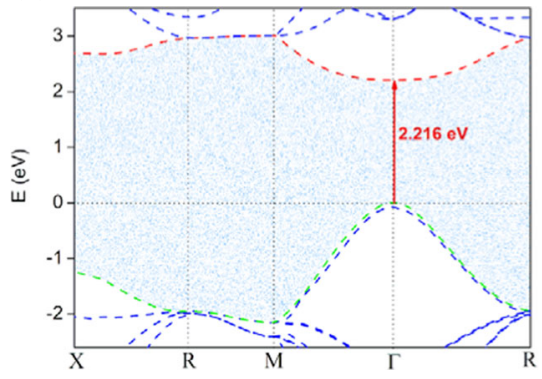

(d)

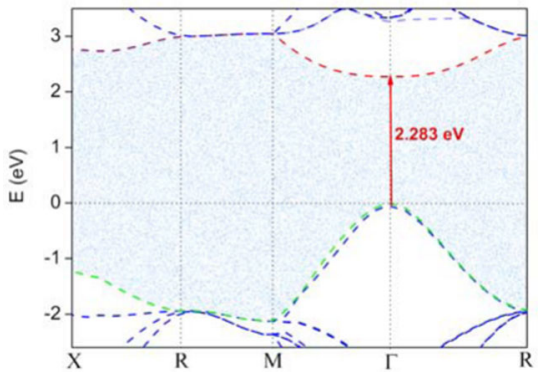

(b)

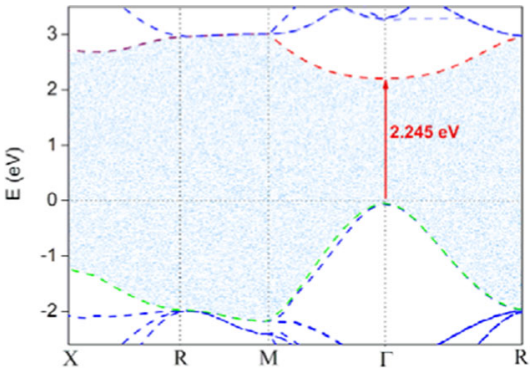

(e)

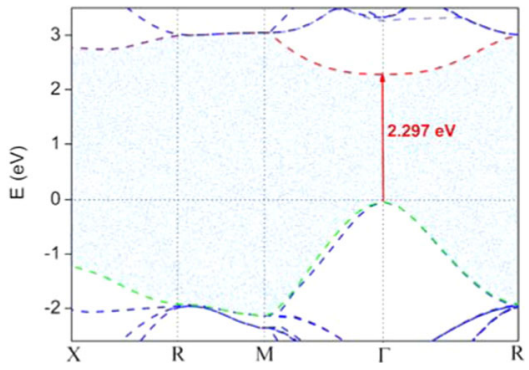

(c)

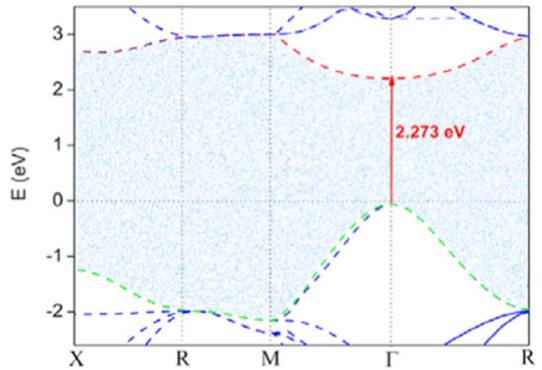

(f)

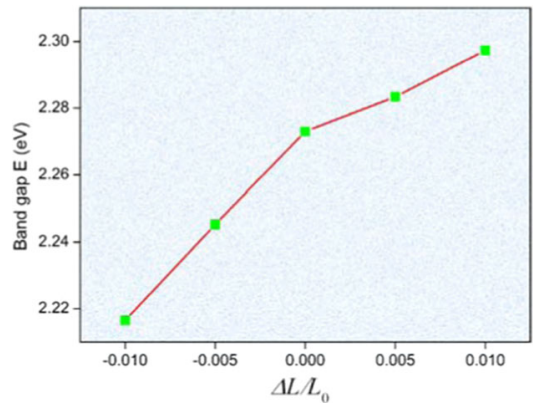

Fig. 5 Band structures of T-carbon under different strain with lattice dilation $\Delta L / L_{0}$ of $\mathbf{a}-0.01, \mathbf{b}-0.005, \mathbf{c} 0$, d 0.005 , and e 0.01 calculated with HSE06 combined with optB86b-vdW interaction. The Fermi level has been shifted to $0 \mathrm{eV}$. The corresponding bandgap has been marked with a red arrow and its value is listed alongside. The bandgap evolution trend under different lattice dilation is shown in $\mathbf{f}$

known to underestimate the bandgap of semiconductors, PBEsol ${ }^{19}$ and hybrid HSE06 functional ${ }^{20}$ methods are also used here to obtain an accurate bandgap based on the structures obtained from the full optimization with the consideration of vdW interactions.

\section{Carrier mobility calculation}

For inorganic semiconductors, the coherent wavelength of thermally activated electrons or holes at room temperature is close to the acoustic photon wavelength and is much larger than their lattice constant. The scattering of the thermal electron or hole can be dominated by the electron-acoustic phonon coupling at low-energy regime, ${ }^{48,56-58}$ which can be calculated by the DP theory proposed by Bardeen and Shockley. ${ }^{18}$ On the basis of effective mass approximation, the carrier mobility $(\mu)$ for 3D materials can be expressed as

$\mu^{3 \mathrm{D}}=\frac{2 \sqrt{2 \pi} e \hbar^{4} C^{3 \mathrm{D}}}{3\left(\kappa_{\mathrm{B}} T\right)^{3 / 2} m^{* 5 / 2} E_{1}^{2}}$,

where $\kappa_{\mathrm{B}}$ is the Boltzmann's constant, $\hbar$ is the Planck's constant, $T$ is the temperature, and $C^{3 \mathrm{D}}$ is the $3 \mathrm{D}$ elastic constant, defined as $C^{3 \mathrm{D}}=\left[\partial^{2} E / \partial \delta^{2}\right] /$ $V_{0}$ with $V_{0}$ being the cell volume at equilibrium, $E$ is the total energy, and $\delta$ is the applied uniaxial strain. $E_{1}$ is the DP constant proportional to the band edge shift induced by applied strain along the transport direction. $E_{1}$ is defined as $\Delta V_{i} /\left(\Delta / / I_{0}\right)$, where $\Delta V_{i}$ is the energy change of the $i$ th band subject to lattice dilation $\Delta / / I_{0}$ along the transport direction (calculated with a step of $0.5 \%) . m^{*}$ is the effective mass in the transport direction and defined as $m^{*}=\hbar^{2}\left(\partial^{2} \varepsilon(k) / \partial k^{2}\right)^{-1}$, where $k$ is the wave-vector magnitude in momentum space. The anisotropic relaxation-time $\tau$ can be calculated from the elastic constant $C^{3 D}$, the DP constant $E_{1}$ and the effective mass $m^{*}$, as defined by $\tau=\mu m^{*} / e$. The temperature used for the calculations of carrier mobility and relaxation-time is at room temperature $(300 \mathrm{~K})$.

\section{DATA AVAILABILITY}

All data generated or analyzed during this study are included in this published article (and its supplementary information files).

\section{ACKNOWLEDGEMENTS}

The authors gratefully acknowledge financial support from Ministry of Education, Singapore (Academic Research Fund TIER 1-RG128/14) and by Economic Development Board of Singapore and Infineon Technologies Asia Pacific Pte Ltd. through the Industrial Postgraduate Programme, Nanyang Technological University, Singapore.

\section{AUTHOR CONTRIBUTIONS}

P.-P.S. and K.Z. conceived and designed the project. P.-P.S. performed the DFT calculations and related analysis. P.-P.S., L.B., D.R.K., and K.Z. analyzed the results. P.-P. S. wrote the manuscript, L.B., D.R.K., and K.Z. helped to revise the manuscript. All authors discussed and commented on the manuscript.

\section{ADDITIONAL INFORMATION}

Supplementary information accompanies the paper on the npj Computational Materials website (https://doi.org/10.1038/s41524-018-0146-z).

Competing interests: The authors declare no competing interests.

Publisher's note: Springer Nature remains neutral with regard to jurisdictional claims in published maps and institutional affiliations.

\section{REFERENCES}

1. Jeon, N. J. et al. Compositional engineering of perovskite materials for highperformance solar cells. Nature 517, 476 (2015).

2. Zhou, H. et al. Interface engineering of highly efficient perovskite solar cells. Science 345, 542-546 (2014).

3. Yang, W. S. et al. High-performance photovoltaic perovskite layers fabricated through intramolecular exchange. Science 348, 1234-1237 (2015).

4. Kojima, A. et al. Organometal halide perovskites as visible-light sensitizers for photovoltaic cells. J. Am. Chem. Soc. 131, 6050-6051 (2009).

5. Best Research-Cell Efficiencies. http://www.nrel.gov/ncpv/images/efficiency_chart. jpg (Accessed March 2018).

6. Moehl, T. et al. Strong photocurrent amplification in perovskite solar cells with a porous $\mathrm{TiO}_{2}$ blocking layer under reverse bias. J. Phys. Chem. Lett. 5, 3931-3936 (2014). 
7. Ravi, V. K. et al. Hierarchical arrays of cesium lead halide perovskite nanocrystals through electrophoretic deposition. J. Am. Chem. Soc. 140, 8887-8894 (2018).

8. Liu, D. \& Kelly, T. L. Perovskite solar cells with a planar heterojunction structure prepared using room-temperature solution processing techniques. Nat. Photonics 8, 133 (2014).

9. You, J. et al. Improved air stability of perovskite solar cells via solution-processed metal oxide transport layers. Nat. Nanotech. 11, 75 (2016).

10. Son, D.-Y. et al. $11 \%$ efficient perovskite solar cell based on $\mathrm{ZnO}$ nanorods: an effective charge collection system. J. Phys. Chem. C 118, 16567-16573 (2014).

11. Zhu, Z. et al. Enhanced efficiency and stability of inverted perovskite solar cells using highly crystalline $\mathrm{SnO}_{2}$ nanocrystals as the robust electron-transporting layer. Adv. Mater. 28, 6478-6484 (2016).

12. Wang, $K$. et al. Low-temperature and solution-processed amorphous $W O_{x}$ as electron-selective layer for perovskite solar cells. J. Phys. Chem. Lett. 6, 755-759 (2015).

13. Wojciechowski, K. et al. $C_{60}$ as an efficient n-type compact layer in perovskite solar cells. J. Phys. Chem. Lett. 6, 2399-2405 (2015).

14. Seo, J. et al. Benefits of very thin PCBM and LiF layers for solution-processed p-i-n perovskite solar cells. Energy Environ. Sci. 7, 2642-2646 (2014).

15. Jeng, J. Y. et al. Nickel oxide electrode interlayer in $\mathrm{CH}_{3} \mathrm{NH}_{3} \mathrm{Pbl}_{3}$ perovskite/PCBM planar-heterojunction hybrid solar cells. Adv. Mater. 26, 4107-4113 (2014).

16. Sheng, X.-L. et al. T-carbon: a novel carbon allotrope. Phys. Rev. Lett. 106, 155703 (2011).

17. Zhang, J. et al. Pseudo-topotactic conversion of carbon nanotubes to T-carbon nanowires under picosecond laser irradiation in methanol. Nat. Commun. 8, 683 (2017).

18. Bardeen, J. \& Shockley, W. Deformation potentials and mobilities in non-polar crystals. Phys. Rev. 80, 72 (1950).

19. Csonka, G. I. et al. Assessing the performance of recent density functionals for bulk solids. Phys. Rev. B 79, 155107 (2009).

20. Heyd, J., Scuseria, G. E. \& Ernzerhof, M. Hybrid functionals based on a screened Coulomb potential. J. Chem. Phys. 118, 8207-8215 (2003).

21. Klimeš, J., Bowler, D. R. \& Michaelides, A. Van der Waals density functionals applied to solids. Phys. Rev. B 83, 195131 (2011).

22. Klimeš, J., Bowler, D. R. \& Michaelides, A. Chemical accuracy for the van der Waals density functional. J. Phys. Condens. Mater. 22, 022201 (2009).

23. Lee, K. et al. Higher-accuracy van der Waals density functional. Phys. Rev. B 82 081101 (2010).

24. Grimme, S. Semiempirical GGA-type density functional constructed with a longrange dispersion correction. J. Comput. Chem. 27, 1787-1799 (2006).

25. Vidal, J. et al. Effects of electronic and lattice polarization on the band structure of delafossite transparent conductive oxides. Phys. Rev. Lett. 104, 136401 (2010).

26. Körner, W. et al. Prediction of subgap states in Zn-and Sn-based oxides using various exchange-correlation functionals. Phys. Rev. B 90, 195142 (2014).

27. Lazar, P. et al. The nature of bonding and electronic properties of graphene and benzene with iridium adatoms. Phys. Chem. Chem. Phys. 16, 20818-20827 (2014).

28. $\mathrm{Li}, \mathrm{T}$. et al. Phase stability and electronic structure of prospective Sb-based mixed sulfide and iodide 3D perovskite $\left(\mathrm{CH}_{3} \mathrm{NH}_{3}\right) \mathrm{SbSl}_{2}$. J. Phys. Chem. Lett. 9, 3829-3833 (2018).

29. Zhou, Z. et al. Interface engineering for high-performance perovskite hybrid solar cells. J. Mater. Chem. A 3, 19205-19217 (2015).

30. Rao, H. S. et al. Improving the extraction of photogenerated electrons with $\mathrm{SnO}_{2}$ nanocolloids for efficient planar perovskite solar cells. Adv. Funct. Mater. 25, 7200-7207 (2015).

31. Yang, D. et al. High efficiency planar-type perovskite solar cells with negligible hysteresis using EDTA-complexed $\mathrm{SnO}_{2}$. Nat. Commun. 9, 3239 (2018).

32. Li, S. et al. Interface engineering of high efficiency perovskite solar cells based on ZnO nanorods using atomic layer deposition. Nano Res. 10, 1092-1103 (2017).

33. Xiong, L. et al. Fully high-temperature-processed $\mathrm{SnO}_{2}$ as blocking layer and scaffold for efficient, stable, and hysteresis-free mesoporous perovskite solar cells. Adv. Funct. Mater. 28, 1706276 (2018).

34. Yang, G. et al. Reducing hysteresis and enhancing performance of perovskite solar cells using low-temperature processed $\mathrm{Y}$-doped $\mathrm{SnO}_{2}$ nanosheets as electron selective layers. Small 13, 1601769 (2017)

35. Choi, K. et al. Thermally stable, planar hybrid perovskite solar cells with high efficiency. Energy Environ. Sci. https://doi.org/10.1039/C8EE02242A (2018).
36. Chou, H.-H. et al. Zinc porphyrin-ethynylaniline conjugates as novel holetransporting materials for perovskite solar cells with power conversion efficiency of $16.6 \%$. ACS Energy Lett. 1, 956-962 (2016).

37. Frisch, M. et al. Gaussian 16, Revision A. 03. (Gaussian Inc., Wallingford CT, 2016)

38. O'regan, B. \& Grätzel, M. A low-cost, high-efficiency solar cell based on dyesensitized colloidal $\mathrm{TiO}_{2}$ films. Nature 353, 737 (1991).

39. Ke, W. et al. Perovskite solar cell with an efficient $\mathrm{TiO}_{2}$ compact film. ACS Appl. Mater. Interfaces 6, 15959-15965 (2014).

40. Burschka, J. et al. Sequential deposition as a route to high-performance perovskite-sensitized solar cells. Nature 499, 316 (2013).

41. Tan, Z.-K. et al. Bright light-emitting diodes based on organometal halide perovskite. Nat. Nanotechnol. 9, 687-692 (2014).

42. Xiong, L. et al. Performance enhancement of high temperature $\mathrm{SnO}_{2}$-based planar perovskite solar cells: electrical characterization and understanding of the mechanism. J. Mater. Chem. A 4, 8374-8383 (2016).

43. Umari, P., Mosconi, E. \& De Angelis, F. Relativistic GW calculations on $\mathrm{CH}_{3} \mathrm{NH}_{3} \mathrm{Pbl}_{3}$ and $\mathrm{CH}_{3} \mathrm{NH}_{3} \mathrm{Snl}_{3}$ perovskites for solar cell applications. Sci. Rep. 4, 4467 (2014).

44. Qiao, J. et al. High-mobility transport anisotropy and linear dichroism in few-layer black phosphorus. Nat. Commun. 5, 4475 (2014).

45. Ma, Y., Kuc, A. \& Heine, T. Single-layer $\mathrm{Tl}_{2} \mathrm{O}$ : a metal-shrouded $2 \mathrm{D}$ semiconductor with high electronic mobility. J. Am. Chem. Soc. 139, 11694-11697 (2017).

46. Murphy-Armando, F. \& Fahy, S. First-principles calculation of carrier-phonon scattering in n-type $\mathrm{Si}_{1-\mathrm{x}} \mathrm{Ge}_{\mathrm{x}}$ alloys. Phys. Rev. B 78, 035202 (2008).

47. Tiwana, $\mathrm{P}$. et al. Electron mobility and injection dynamics in mesoporous $\mathrm{ZnO}$, $\mathrm{SnO}_{2}$, and $\mathrm{TiO}_{2}$ films used in dye-sensitized solar cells. ACS Nano 5, 5158-5166 (2011).

48. Cai, Y., Zhang, G. \& Zhang, Y.-W. Polarity-reversed robust carrier mobility in monolayer $\mathrm{MoS}_{2}$ nanoribbons. J. Am. Chem. Soc. 136, 6269-6275 (2014).

49. Oga, $\mathrm{H}$. et al. Improved understanding of the electronic and energetic jandscapes of perovskite solar cells: high local charge carrier mobility, reduced recombination, and extremely shallow traps. J. Am. Chem. Soc. 136, 13818-13825 (2014).

50. Motta, C., El-Mellouhi, F. \& Sanvito, S. Charge carrier mobility in hybrid halide perovskites. Sci. Rep. 5, 12746 (2015).

51. Yang, D. et al. Surface optimization to eliminate hysteresis for record efficiency planar perovskite solar cells. Energy Environ. Sci. 9, 3071-3078 (2016)

52. Perdew, J. P., Burke, K. \& Ernzerhof, M. Generalized gradient approximation made simple. Phys. Rev. Lett. 77, 3865 (1996).

53. Blöchl, P. E. Projector augmented-wave method. Phys. Rev. B 50, 17953 (1994).

54. Román-Pérez, G. \& Soler, J. M. Efficient implementation of a van der Waals density functional: application to double-wall carbon nanotubes. Phys. Rev. Lett. 103, 096102 (2009).

55. Dion, M. et al. Van der Waals density functional for general geometries. Phys. Rev. Lett. 92, 246401 (2004).

56. Kaasbjerg, K., Thygesen, K. S. \& Jacobsen, K. W. Phonon-limited mobility in n-type single-layer $\mathrm{MoS}_{2}$ from first principles. Phys. Rev. B 85, 115317 (2012).

57. Kaasbjerg, K., Thygesen, K. S. \& Jauho, A.-P. Acoustic phonon limited mobility in two-dimensional semiconductors: deformation potential and piezoelectric scattering in monolayer $\mathrm{MoS}_{2}$ from first principles. Phys. Rev. B 87, 235312 (2013).

58. Long, M.-Q. et al. Theoretical predictions of size-dependent carrier mobility and polarity in graphene. J. Am. Chem. Soc. 131, 17728-17729 (2009).

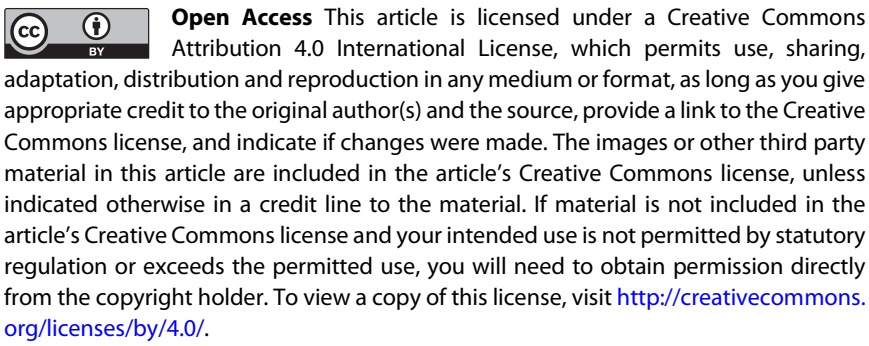

(c) The Author(s) 2019 